Pacific

Journal of

Mathematics

PURE-PERIODIC MODULES AND A STRUCTURE OF PURE-PROJECTIVE RESOLUTIONS

DANiEL Simson 


\title{
PURE-PERIODIC MODULES AND A STRUCTURE OF PURE-PROJECTIVE RESOLUTIONS
}

\author{
DANiEL Simson
}

Dedicated to Stanistaw Balcerzyk on the occasion of his seventieth birthday

We investigate the structure of pure-syzygy modules in a pure-projective resolution of any right $R$-module over an associative ring $R$ with an identity element. We show that a right $R$-module $M$ is pure-projective if and only if there exists an integer $n \geq 0$ and a pure-exact sequence $0 \rightarrow M \rightarrow P_{n} \rightarrow$ $\cdots \rightarrow P_{0} \rightarrow M \rightarrow 0$ with pure-projective modules $P_{n}, \ldots, P_{0}$. As a consequence we get the following version of a result in Benson and Goodearl, 2000: A flat module $M$ is projective if $M$ admits an exact sequence $0 \rightarrow M \rightarrow F_{n} \rightarrow \cdots \rightarrow F_{0} \rightarrow$ $M \rightarrow 0$ with projective modules $F_{n}, \ldots, F_{0}$.

\section{Introduction.}

Throughout this paper $R$ is an associative ring with an identity element. We denote by $\operatorname{Mod}(R)$ the category of all right $R$-modules. We recall (see [12]) that an exact sequence $\cdots \rightarrow X_{n-1} \rightarrow X_{n} \rightarrow X_{n+1} \rightarrow \ldots$ in $\operatorname{Mod}(R)$ is said to be pure (in the sense of Cohn [6]) if the induced sequence $\cdots \rightarrow$ $X_{n-1} \otimes_{R} L \rightarrow X_{n} \otimes_{R} L \rightarrow X_{n+1} \otimes_{R} L \rightarrow \ldots$ of abelian groups is exact for any left $R$-module $L$. An epimorphism $f: Y \longrightarrow Z_{f}$ in $\operatorname{Mod}(R)$ is said to be pure if the exact sequence $0 \longrightarrow \operatorname{Ker} f \longrightarrow Y \stackrel{f}{\longrightarrow} Z \longrightarrow 0$ is pure. A submodule $X$ of a right $R$-module $Y$ is said to be pure if the exact sequence $0 \rightarrow X \rightarrow Y \rightarrow Y / X \rightarrow 0$ is pure. A module $P$ in $\operatorname{Mod}(R)$ is said to be pure-projective if for any pure epimorphism $f: Y \longrightarrow Z$ in $\operatorname{Mod}(R)$ the induced group homomorphism $\operatorname{Hom}_{R}(P, f): \operatorname{Hom}_{R}(P, Y) \longrightarrow \operatorname{Hom}_{R}(P, Z)$ is surjective. The following facts are well-known (see [14], [15], [29], [31]):

(i) A module $P$ in $\operatorname{Mod}(R)$ is pure-projective if and only if $P$ is a direct summand of a direct sum of finitely presented modules.

(ii) Every module $M$ in $\operatorname{Mod}(R)$ admits a pure-projective pure resolution $\mathbf{P}_{*}$ in $\operatorname{Mod}(R)$, that is, there is a pure-exact sequence

$$
\ldots \longrightarrow P_{n} \stackrel{d_{n}}{\longrightarrow} P_{n-1} \stackrel{d_{n-1}}{\longrightarrow} \ldots \stackrel{d_{2}}{\longrightarrow} P_{1} \stackrel{d_{1}}{\longrightarrow} P_{0} \stackrel{d_{0}}{\longrightarrow} M \rightarrow 0
$$

where the modules $P_{0}, \ldots, P_{n}, \ldots$ are pure-projective.

The main results of the paper are the following two theorems: 
Theorem 1.2. Let $M$ be a right $R$-module and (1.1) a pure-exact sequence in $\operatorname{Mod}(R)$ such that the modules $P_{0}, \ldots, P_{n}, \ldots$ are pure-projective. Then, for each $n \geq 0$, the $n$-th pure-syzygy module Ker $d_{n}$ of $M$ is an $\aleph_{n}$-directed union of $\aleph_{n}$-generated pure-projective $R$-modules, which are pure submodules of $P_{n}$ and of $\operatorname{Ker} d_{n}$.

Theorem 1.3. Let $M$ be a right $R$-module. If there exists a pure-exact sequence

$$
0 \rightarrow M \longrightarrow P_{n} \longrightarrow P_{n-1} \longrightarrow \ldots \longrightarrow P_{1} \longrightarrow P_{0} \longrightarrow M \rightarrow 0
$$

in $\operatorname{Mod}(R)$ such that the modules $P_{0}, \ldots, P_{n}$ are pure-projective, then $M$ is pure-projective.

In other words, every pure-periodic $R$-module $M$ is pure-projective. As a consequence of Theorem 1.3 we get the following version of a recent result by Benson and Goodearl in [5].

Corollary 1.4. Let $M$ be a right flat $R$-module. If there exists an exact sequence

$$
0 \rightarrow M \longrightarrow F_{n} \longrightarrow F_{n-1} \longrightarrow \ldots \longrightarrow F_{1} \longrightarrow F_{0} \longrightarrow M \rightarrow 0
$$

in $\operatorname{Mod}(R)$ such that the modules $F_{0}, \ldots, F_{n}$ are projective, then $M$ is projective.

Our Theorem 1.2 extends the main projective resolution structure theorem [23, Theorem 1.5] (see also [17, Theorem 3.3] for $n=0$ ) from flat modules to arbitrary modules. The Corollary 1.4 with $n=0$ coincides with [5, Theorem 2.5].

The main results of the paper are proved in Sections 3 and 4. In Section 2 we collect preliminary facts and notation we need throughout the paper. In Section 5 we show that Theorems 1.2 and 1.3 remain valid in any locally finitely presented Grothendieck category $\mathcal{A}$.

Throughout this paper we use freely the module theory terminology and notation introduced in [1] and [12]. The reader is referred to [8], [12], $[\mathbf{1 4}],[\mathbf{1 6}],[\mathbf{2 6}],[\mathbf{2 9}],[\mathbf{3 1}]$ and to the expository papers $[\mathbf{9}]$ and [28] for a basic background and historical comments on purity and pure homological dimensions.

\section{Preliminaries on the pure-projective dimension.}

We start this section by collecting basic definitions, notation and elementary facts we need throughout this paper.

Given right $R$-modules $M$ and $N$ the $n$-th pure extension group $\operatorname{Pext}_{R}^{n}(M, N)$ is defined to be the $n$-th cohomology group of the complex $\operatorname{Hom}_{R}\left(\mathbf{P}_{*}, N\right)$, where $\mathbf{P}_{*}$ is a pure-projective resolution of $M$ in $\operatorname{Mod}(R)$. 
The pure-projective dimension P.pd $M$ of $M$ is defined to be the minimal integer $m \geq 0$ (or infinity) such that $\operatorname{Pext}_{R}^{m}(M,-)=0$. The right pure global dimension r.P.gl.dim $R$ of $R$ is defined to be the minimal integer $n \geq 0$ (or infinity) such that $\operatorname{Pext}_{R}^{n}=0$. Following [27] we call the ring $R$ right pure semisimple if r.P.gl.dim $R=0$.

The left pure global dimension l.P.gl.dim $R$ of a ring $R$ was introduced in 1967 by R. Kiełpiński [14] and in 1970 by P. Griffith [7]. It was shown in [26, Theorem 2.12] that the right pure global dimension r.P.gl.dim $R$ of the ring $R$ is the supremum of P.pd $M$, where $M$ runs through all right $R$-modules $M$ such that P.pd $M$ is finite. This means that the right finitistic pure global dimension of $R$ and the right pure global dimension of $R$ coincide.

Throughout this paper we denote by $\aleph$ an infinite cardinal number and by $\aleph_{0}$ the cardinality of a countable set. A right $R$-module $M$ is said to be $\aleph$-generated if it is generated by a set of cardinality $\aleph$, and $M$ is $\aleph$-presented if $M$ is $\aleph$-generated and for any epimorphism $f: L \rightarrow M$ with $\aleph$-generated module $L$ the kernel Ker $f$ is $\aleph$-generated, or equivalently, $M$ is a limit of a direct system $\left\{M_{j}, h_{i j}\right\}$ of cardinality $\aleph$ consisting of finitely presented modules $M_{j}$ (see [18], [20], [22], [26]). We say that $M$ is an $\aleph$-directed union of submodules $M_{j}, j \in J$, if for each subset $J_{0}$ of $J$ of cardinality there exist $j_{0} \in J$ such that $M_{t} \subseteq M_{j_{0}}$ for all $t \in J_{0}$.

A union $\bigcup_{\xi<\gamma} M_{\xi}$ of submodules $M_{\xi}$ of $M$ is well-ordered and continuous if $\gamma$ is an ordinal number, $M_{0}=(0), M_{\xi} \subseteq M_{\eta}$ for $\xi<\eta<\gamma$, and $M_{\tau}=\bigcup_{\xi<\tau} M_{\xi}$ for any limit ordinal number $\tau \leq \gamma$ (see [16]).

The following pure version of the well-known Auslander result [2, Proposition 3] is of importance.

Proposition 2.1. Assume that the right $R$-module $M$ is a continuous wellordered union of submodules $M_{\xi}$, with $\xi<\gamma$, where $\gamma$ is an ordinal number. If P.pd $M_{\xi+1} / M_{\xi} \leq m$ for all $\xi<\gamma$, then P.pd $M \leq m$.

Proof. The arguments of Auslander in the proof of [2, Proposition 3] generalise to our situation (see [16, Proposition 1.2]).

We also need the following pure version of the well-known Osofsky result [19] (see also [3]) proved in [16, Corollary 1.4], [8], and in [26, Theorem 2.12 ] in a general context of Grothendieck categories.

Proposition 2.2. Assume that $m \geq 0$ is an integer and $M$ is an arbitrary $\aleph_{m}$-presented right $R$-module. Then P.pd $M \leq m+1$.

By applying the definition of a pure submodule one proves the following useful criterion:

Lemma 2.3. Assume that $P$ is a pure-projective right $R$-module and let $K$ be a submodule of $P$. The following conditions are equivalent: 
(a) $K$ is a pure submodule of $P$.

(b) For any finitely generated submodule $X$ of $K$ there exists an $R$-homomorphism $\varphi: P \rightarrow K$ such that $\operatorname{Im} \varphi$ is contained in a finitely generated $R$-submodule of $K$ and $\left.\varphi\right|_{X}=\operatorname{id}_{X}$.

(c) For any finitely generated submodule $X$ of $K$ there exists an $R$-homomorphism $\varphi: P \rightarrow K$ such that $\left.\varphi\right|_{X}=\operatorname{id}_{X}$.

Proof. Since the module $P$ is pure projective, there exists a module $P^{\prime}$ such that $P \oplus P^{\prime}$ is a direct sum of finitely presented modules. Assume that $K$ is a submodule of $P$ and let $u: K \rightarrow P$ be the embedding.

(a) $\Rightarrow$ (b) Assume that $u: K \rightarrow P$ is a pure monomorphism and $X$ is a finitely generated submodule of $K$. Then the monomorphism $(u, 0)$ : $K \longrightarrow P \oplus P^{\prime}$ is pure and there exists a finitely presented direct summand $L$ of $P \oplus P^{\prime}$ such that $(u, 0)(X) \subseteq L$. Consider the commutative diagram

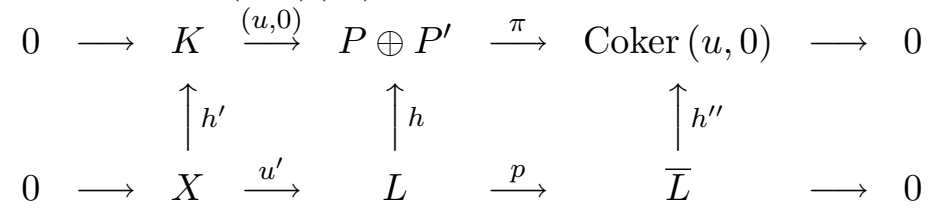

with exact rows, where $h^{\prime}$ is the embedding of $X$ into $K, h$ is a direct summand embedding, $\pi$ is a pure epimorphism and the module $\bar{L}$ is finitely presented. It follows that there exists $v^{\prime \prime} \in \operatorname{Hom}_{R}\left(\bar{L}, P \oplus P^{\prime}\right)$ such that $\pi v^{\prime \prime}=h^{\prime \prime}$, and consequently there exists $v^{\prime} \in \operatorname{Hom}_{R}(L, K)$ such that $v^{\prime} u^{\prime}=$ $h^{\prime}$. Let $\varphi^{\prime}: P \oplus P^{\prime} \longrightarrow K$ be an extension of $v^{\prime}$ to $P \oplus P^{\prime}$ such that $v^{\prime}=\varphi^{\prime} h$ and $\operatorname{Im} \varphi^{\prime}$ is finitely generated. Let $\varphi: P \rightarrow K$ be the restriction of $\varphi^{\prime}$ to $P$. It follows that $\operatorname{Im} \varphi$ is contained in the finitely generated $R$-submodule $\operatorname{Im} \varphi^{\prime}$ of $K$ and, for any $x \in X$, we have $x=h^{\prime}(x)=v^{\prime} u^{\prime}(x)=\varphi^{\prime} h u^{\prime}(x)=$ $\varphi^{\prime}\left(h^{\prime}(x), 0\right)=\varphi^{\prime}(x, 0)=\varphi(x)$. This shows that $\left.\varphi\right|_{X}=\operatorname{id}_{X}$ and (b) follows.

The implication (b) $\Rightarrow$ (c) is obvious.

(c) $\Rightarrow$ (a) Assume that, for any finitely generated submodule $X$ of $K$, there exists an $R$-homomorphism $\varphi: P \rightarrow K$ such that $\left.\varphi\right|_{X}=\operatorname{id}_{X}$. We shall prove that $K$ is a pure submodule of $P$ by showing that the canonical epimorphism $\pi: P \longrightarrow P / K$ is pure. Let $f: L \longrightarrow P / K$ be a homomorphism from a finitely presented module $L$ to $P / K$. Then $L \cong F / N$, where $F$ is a finitely generated free module and $N$ is a finitely generated submodule of $F$. It is clear that there exists a commutative diagram

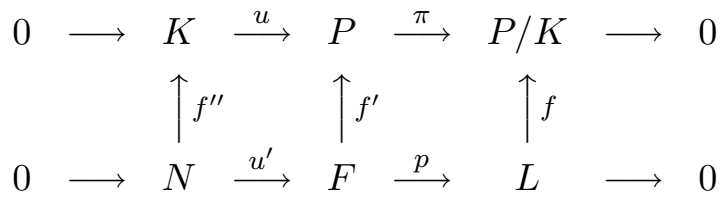

with exact rows, where $p$ is the canonical epimorphism and $u^{\prime}$ is the canonical embedding. Then $X=f^{\prime \prime}(N)$ is a finitely generated submodule of $K$ and, 
according to our assumption, there exits an $R$-homomorphism $\varphi: P \rightarrow K$ such that $\left.\varphi\right|_{X}=\operatorname{id}_{X}$. Note that the homomorphism $v^{\prime}=\varphi f^{\prime}: F \longrightarrow K$ satisfies the equality $f^{\prime \prime}=v^{\prime} u^{\prime}$. It follows that there exists $v^{\prime \prime} \in \operatorname{Hom}_{R}(L, P)$ such that $\pi v^{\prime \prime}=f$. This shows that $\pi$ is a pure epimorphism and finishes the proof of the lemma.

Let $P$ be a pure-projective right $R$-module and let $K$ be a pure submodule of $P$. Following [23, Proposition 1.4] and [24] we define a pure-closure $L^{\diamond}$ of any $R$-submodule $L$ of $K$ as follows. Set $L_{0}=L$ and fix a set $L^{\prime}$ of generators of $L$. By Lemma 2.3, for any finite subset $\lambda$ of $L^{\prime}$ we find an $R$-homomorphism $\varphi_{\lambda}: P \rightarrow K$ such that $\operatorname{Im} \varphi_{\lambda}$ is contained in a finitely generated $R$-submodule $K_{\lambda}$ of $K$, and $\left.\varphi_{\lambda}\right|_{\lambda}=\operatorname{id}_{\lambda}$. Let $L_{1}$ be the $R$-submodule of $L$ generated by the set $L^{\prime \prime}=\bigcup_{\lambda \subseteq L^{\prime}} K_{\lambda}$, where $\lambda$ runs over all finite subsets of $L^{\prime}$. It is clear that $L=L_{0} \subseteq L_{1}$ and, for any finitely generated submodule $X$ of $L_{0}=L$, there exists an $R$-homomorphism $\varphi: P \rightarrow L_{1}$ such that $\operatorname{Im} \varphi$ is contained in a finitely generated $R$-submodule of $L_{1}$, and $\left.\varphi\right|_{X}=\mathrm{id}_{X}$. By choosing a set $L_{1}^{\prime}$ of generators of $L_{1}$ and applying the procedure above with $L^{\prime}$ and $L_{1}^{\prime}$ interchanged, we construct a submodule $L_{2}$ containing $L_{1}$ such that for any finitely generated submodule $X$ of $L_{1}$ there exists an $R$-homomorphism $\varphi: P \rightarrow L_{2}$ such that $\operatorname{Im} \varphi$ is contained in a finitely generated $R$-submodule of $L_{2}$, and $\left.\varphi\right|_{X}=\mathrm{id}{ }_{X}$. Continuing this way we define an ascending sequence

$$
L=L_{0} \subseteq L_{1} \subseteq L_{2} \subseteq \cdots \subseteq L_{m} \subseteq L_{m+1} \subseteq \cdots
$$

of $R$-submodules of $K$, and sets $L_{0}^{\prime}, L_{1}^{\prime}, L_{2}^{\prime}, \ldots L_{m}^{\prime}, L_{m+1}^{\prime} \ldots$ of their generators in such a way that, for each $m \geq 0$ and for any finitely generated submodule $X$ of $L_{m}$, there exists an $R$-homomorphism $\varphi: P \rightarrow L_{m+1}$ such that $\operatorname{Im} \varphi$ is contained in a finitely generated $R$-submodule of $L_{m+1}$, and $\left.\varphi\right|_{X}=\mathrm{id}_{X}$. By Lemma 2.3, the submodule

$$
L^{\diamond}=\bigcup_{m=0}^{\infty} L_{m}
$$

of $K$ is a pure submodule of $P$ (and of $K$ ), and we call it a pure-closure of the $R$-submodule $L$ of $K$. It is clear that $L^{\diamond}$ is not determined uniquely by $L$ and depends on the choice of the modules $K_{\lambda}$, the sets $L_{0}^{\prime}, L_{1}^{\prime}, L_{2}^{\prime}, \ldots L_{m}^{\prime}$, $L_{m+1}^{\prime} \cdots$ and the $R$-homomorphisms $\varphi_{\lambda}: P \rightarrow K$. However, if $\aleph$ is an infinite cardinal number and the module $L$ is $\aleph$-generated then the sets $L_{0}^{\prime}, L_{1}^{\prime}, L_{2}^{\prime}, \ldots L_{m}^{\prime}, L_{m+1}^{\prime} \ldots$ can be chosen of cardinality $\aleph$ and we get the following result.

Lemma 2.5. Assume that $P$ is a pure-projective right $R$-module, $K$ a pure submodule of $P$ and $L$ an $\aleph$-generated submodule of $K$, where $\aleph$ is an infinite 
cardinal number. Then there exists an $\aleph$-generated submodule $L^{\diamond}$ of $K$ such that $L \subseteq L^{\diamond}$ and $L^{\diamond}$ is a pure submodule of $P($ and of $K)$.

We also need the following technical result.

Lemma 2.6. Assume that $\aleph$ is an infinite cardinal number, $h: P \rightarrow K$ is a pure epimorphism in $\operatorname{Mod}(R), P$ is an $\aleph$-generated pure-projective module and $K$ is a pure submodule of a pure-projective module.

(a) The module $K$ has a directed union form $K=\bigcup_{\lambda \in \Omega} K_{\lambda}$, where $\Omega$ is a set of cardinality $\leq \aleph$ and $K_{\lambda}$ is a countably generated pure-projective pure submodule of $K$, for each $\lambda \in \Omega$.

(b) The module Ker $h$ is $\aleph$-generated.

Proof. Let $h: P \rightarrow K$ be a pure-epimorphism. We set $L=\operatorname{Ker} h$ and assume that the module $P$ is $\aleph$-generated. Then there exist a set $\Omega$ of cardinality $\leq \aleph$ and a family of finitely generated submodules $P_{\lambda}$ of $P$, with $\lambda \in \Omega$, such that $P=\bigcup_{\lambda \in \Omega} P_{\lambda}$ is a directed union. By our assumption, $K$ is a pure submodule of a pure-projective module $P_{0}$. Let $P_{0}^{\prime}$ be a right $R$-module such that $P_{0} \oplus P_{0}^{\prime}$ is a direct sum of finitely presented modules.

For each $\lambda \in \Omega$, we consider the commutative diagram

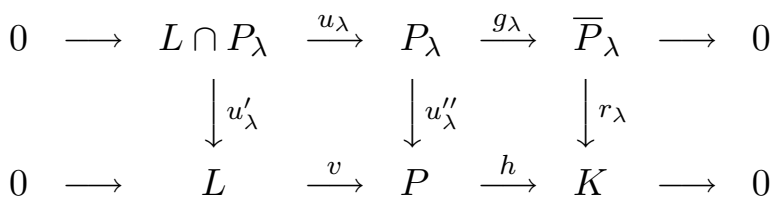

with exact rows, where $\bar{P}_{\lambda}=P_{\lambda} / L \cap P_{\lambda}, u_{\lambda}, u_{\lambda}^{\prime}, u_{\lambda}^{\prime \prime}, v$ are the embeddings and $r_{\lambda}$ is the natural $R$-module homomorphism induced by $u_{\lambda}^{\prime \prime}$. Since $V_{\lambda}=$ $\operatorname{Im} r_{\lambda}=h\left(P_{\lambda}\right)$ is a finitely generated submodule of $K$ and $K$ is a pure submodule of $P_{0} \oplus P_{0}^{\prime}$ then $V_{\lambda}$ is $\aleph_{0}$-generated and, according to Lemma 2.5, there exists an $\aleph_{0}$-generated pure submodule $V_{\lambda}^{\diamond}$ of $P_{0} \oplus P_{0}^{\prime}$ contained in $K$ and containing $V_{\lambda}$. It follows that $V_{\lambda}^{\diamond}$ is a pure submodule of an $\aleph_{0^{-}}$ generated direct summand $P^{\prime}$ of $P_{0} \oplus P_{0}^{\prime}$. Then the module $P^{\prime} / V_{\lambda}^{\diamond}$ is $\aleph_{0^{-}}$ presented and Proposition 2.2 yields (see also [10])

$$
\text { P.pd } P^{\prime} / V_{\lambda}^{\diamond} \leq 1 \text {. }
$$

It follows that the submodule $V_{\lambda}^{\diamond}$ of $K$ is pure-projective. If we set $K_{\lambda}=$ $V_{\lambda}^{\diamond}$, then obviously $K=\bigcup_{\lambda \in \Omega} K_{\lambda}$ is a directed union and $K_{\lambda}$ is a countably generated pure-projective pure submodule of $K$ for each $\lambda \in \Omega$. This proves Statement (a).

Since the epimorphism $h: P \rightarrow K$ is pure, the embedding $w_{\lambda}: V_{\lambda}^{\diamond} \hookrightarrow K$ extends to an $R$-module homomorphism $f_{\lambda}: V_{\lambda}^{\diamond} \longrightarrow P$ such that $h f_{\lambda}=$ 
$w_{\lambda}$. Then the composed $R$-module homomorphism $\psi_{\lambda}=f_{\lambda} r_{\lambda}: \bar{P}_{\lambda} \longrightarrow P$ satisfies $h \psi_{\lambda}=r_{\lambda}$ and, by the commutativity of the diagram (2.7), there exists an $R$-module homomorphism $\varphi_{\lambda}: P_{\lambda} \rightarrow L$ such that $\varphi_{\lambda} u_{\lambda}=u_{\lambda}^{\prime}$. Hence we easily conclude that $L=\sum_{\lambda \in \Omega} \operatorname{Im} \varphi_{\lambda}$ and therefore $L$ is $\aleph$-generated, because $|\Omega| \leq \aleph$ and $\operatorname{Im} \varphi_{\lambda}$ is finitely generated for any $\lambda \in \Omega$. This finishes the proof.

\section{A pure-projective structure of pure-syzygy modules.}

The aim of this section is to prove Theorem 1.2 on the pure-projective structure of the $n$-th pure-syzygy module of any right $R$-module $M$, that is, the pure submodule $\operatorname{Ker} d_{n}$ of $P_{n}$ in a pure-projective resolution (1.1) of $M$.

We start with the following key proposition.

Proposition 3.1. Assume that $R$ is a ring, $\aleph$ is an infinite cardinal number, $M$ is a right $R$-module, $n \geq 0$ an integer and

$$
0 \rightarrow K_{n} \longrightarrow P_{n} \stackrel{d_{n}}{\longrightarrow} P_{n-1} \stackrel{d_{n-1}}{\longrightarrow} \ldots \stackrel{d_{2}}{\longrightarrow} P_{1} \stackrel{d_{1}}{\longrightarrow} P_{0} \stackrel{d_{0}}{\longrightarrow} M \rightarrow 0
$$

is a pure-exact sequence, where $K_{n}=\operatorname{Ker} d_{n}$ and the modules $P_{0}, \ldots, P_{n}$ are pure-projective.

(a) For any $\aleph$-generated submodule $N$ of $K_{n}$ and any $\aleph$-generated submodule $L$ of $K_{0}=$ Ker $d_{0}$ there exist an $\aleph$-generated pure submodule $N$ of $P_{n}$, and $\aleph$-generated pure submodule $L$ of $P_{0}$, an $\aleph-$ generated direct summands $P_{1}^{\prime}, \ldots, P_{n}^{\prime}$ of $P_{1}, \ldots, P_{n}$, respectively, such that $d_{j}\left(P_{j}^{\prime}\right) \subseteq P_{j-1}^{\prime}$ for $j=1, \ldots, n, N \subseteq N{ }_{n} \subseteq K_{n}=$ Ker $d_{n}$, $L \subseteq L \subseteq K_{0}=\operatorname{Ker} d_{0}$, and, for each $n \geq 1$, the sequence

$$
0 \rightarrow N^{\star} \longrightarrow P_{n}^{\prime} \stackrel{d_{n}^{\prime}}{\longrightarrow} P_{n-1}^{\prime} \stackrel{d_{n-1}^{\prime}}{\longrightarrow} \ldots \stackrel{\bar{d}_{2}^{\prime}}{\longrightarrow} P_{1}^{\prime} \stackrel{d_{1}^{\prime}}{\longrightarrow} L^{\bullet_{0}} \rightarrow 0
$$

is pure-exact, where $d_{j}^{\prime}$ is the restriction of $d_{j}$ to $P_{j}^{\prime}$. In case $n=0$ we have $N^{{ }_{0}}=L^{\star}$.

(b) If $n \geq 1$ and there is an $R$-module isomorphism $K_{n} \cong K_{0}$, then there exists a pure-exact sequence $(* *)$ satisfying the conditions listed in (a) and such that $N{ }^{n} \cong L^{*}$.

Proof. (a) Since any pure-projective module is a direct summand of a direct sum of finitely presented modules then, according to the well-known Kaplansky theorem [13], there are pairwise disjoint sets $I_{0}, I_{1}, \ldots, I_{n}$ and countably generated pure-projective modules $Q_{t}$, with $t \in I_{0} \cup I_{1} \cup \cdots \cup I_{n}$, such that, for each for $j \in\{0,1, \ldots, n\}$, the pure-projective module $P_{j}$ in $(*)$ has the form

$$
P\left(I_{j}\right)=\bigoplus_{t \in I_{j}} Q_{t},
$$

up to isomorphism. Without loss of generality we can suppose that $P_{j}=$ $P\left(I_{j}\right)$ for $j=0,1, \ldots, n$. 
Instead of the statement (a) we prove inductively on $n \geq 0$, like $[\mathbf{2 3}$, Proposition 1.4], the following stronger form of (a).

Assume that, for each $j \in\{0,1, \ldots, n\}$, the pure-projective module $P_{j}$ in (*) has the form $P\left(I_{j}\right)$ as above. Then the following two statements hold.

(a1) For any $\aleph$-generated submodule $N$ of $K_{n}$ and any $\aleph$-generated submodule $L$ of $K_{0}=\operatorname{Ker} d_{0}$ there exist an $\aleph$-generated pure submodule $N$ of $P_{n}=P\left(I_{n}\right)$, $\aleph$-generated pure submodule $L$ of $P_{0}=P\left(I_{0}\right)$, and subsets $I_{0}^{\prime}, \ldots, I_{n}^{\prime}$ of $I_{0}, \ldots, I_{n}$, respectively, of cardinality $\leq \aleph$ such that $d_{j}\left(P\left(I_{j}^{\prime}\right)\right) \subseteq P\left(I_{j-1}^{\prime}\right)$ for $j=1, \ldots, n, N \subseteq N^{*} \subseteq K_{n}=$ Ker $d_{n}$, $L \subseteq L \subseteq K_{0}=\operatorname{Ker} d_{0}$, and, for each $n \geq 1$, the sequence (**) is pure-exact, where $P_{j}^{\prime}=P\left(I_{j}^{\prime}\right)$ and $d_{j}^{\prime}$ is the restriction of $d_{j}$ to $P_{j}^{\prime}$. In case $n=0$ we have $N=L$.

(a2) Assume that $N, L, N{ }^{\star}, L$ and $I_{0}^{\prime}, \ldots, I_{n}^{\prime}$ are such that the statement (a1) holds, and let $N^{\prime}$ and $L^{\prime}$ be $\aleph$-generated submodules of $K_{n}$ and $K_{0}$ containing $N$ and $L$, respectively. Then there exist an $\aleph$-generated pure submodule $N^{\prime}$ of $P_{n}=P\left(I_{n}\right)$, ※-generated pure submodule $L^{\prime{ }_{0}}$ of $P_{0}=P\left(I_{0}\right)$ and subsets $I_{0}^{\prime \prime}, \ldots, I_{n}^{\prime \prime}$ of $I_{0}, \ldots, I_{n}$, respectively, of cardinality $\leq \aleph$ such that $d_{j}\left(P\left(I_{j}^{\prime \prime}\right)\right) \subseteq P\left(I_{j-1}^{\prime \prime}\right)$ for $j=1, \ldots, n$, $N^{\prime} \subseteq N^{\prime}{ }_{n} \subseteq K_{n}, L^{\prime} \subseteq L^{\prime{ }_{0}} \subseteq K_{0}, N^{{ }_{n}} \subseteq N^{\prime{ }_{n}}, L^{{ }_{n}} \subseteq L^{\prime{ }_{0}}$, the diagram

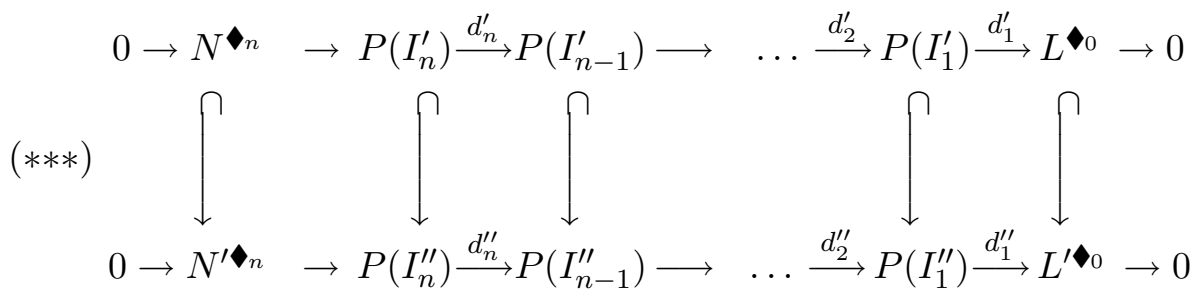

is commutative and has pure-exact rows, where the vertical arrows are natural embeddings induced by the inclusions $I_{0}^{\prime} \subseteq I_{0}^{\prime \prime}, \ldots, I_{n}^{\prime} \subseteq I_{n}^{\prime \prime}$ and $d_{j}^{\prime \prime}$ is the restriction of $d_{j}$ to $P\left(I_{j}^{\prime \prime}\right)$ for $j=1, \ldots, n$. In case $n=0$ we have $N^{\prime}=L^{\prime{ }_{0}}$.

Assume that $n=0$. Since the submodules $N$ and $L$ of $K_{0}$ are $\aleph$-generated then applying Lemma 2.5 to the $\aleph$-generated submodule $N+L$ of $K_{0}$ we get an $\aleph$-generated pure submodule $(N+L)^{\diamond}$ of $P\left(I_{0}\right)$ and of $K_{0}$. It follows that there is a subset $I_{0}^{\prime}$ of $I_{0}$ of cardinality $\leq \aleph$ such that $(N+L)^{\diamond}$ is a pure submodule of $P\left(I_{0}^{\prime}\right) \subseteq P\left(I_{0}\right)$. If we set $N N^{\diamond}=L^{\diamond}=(N+L)^{\diamond}$ we get (a1). Statement (a2) follows in a similar way.

Assume that $n \geq 1$. First we prove the following:

Claim. For any $\aleph$-generated submodule $Y$ of $K_{n-1}$ and any subset $\mathcal{Y}$ of $I_{n}$ of cardinality $\leq \aleph$ there exists an $\aleph^{\aleph}$-generated pure submodule $Y^{\prime}$ of $K_{n-1}$ 
containing $Y$ and a subset $\mathcal{Y}^{\prime}$ of $I_{n}$ of cardinality $\leq \aleph$ containing $\mathcal{Y}$ such that:

(c1) $d_{n}\left(P\left(\mathcal{Y}^{\prime}\right)\right)=Y^{\prime}$

(c2) the restriction $d_{n}^{\prime}: P\left(\mathcal{Y}^{\prime}\right) \longrightarrow Y^{\prime}$ of $d_{n}$ to $P\left(\mathcal{Y}^{\prime}\right)$ is a pure epimorphism, and

(c3) the submodule $\operatorname{Ker} d_{n}^{\prime}$ of $P\left(\mathcal{Y}^{\prime}\right)$ is $\aleph$-generated.

Let $Y$ be an $\aleph$-generated submodule of $K_{n-1}$ and $\mathcal{Y}$ a subset of $I_{n}$ of cardinality $\leq \aleph$. We construct the subset $\mathcal{Y}^{\prime}$ of $I_{n}$ as the union $\mathcal{Y}^{\prime}=\bigcup_{j=1}^{\infty} \mathcal{Y}_{j}$ of subsets

$$
\mathcal{Y} \subseteq \mathcal{Y}_{1} \subseteq \mathcal{Y}_{2} \subseteq \cdots \subseteq \mathcal{Y}_{j} \subseteq \mathcal{Y}_{j+1} \subseteq \cdots
$$

of $I_{n}$ of cardinality $\leq \aleph$, and the module $Y^{\prime}$ as the union $Y^{\prime}=\bigcup_{j=1}^{\infty} Y^{(j)}$ of $\aleph$-generated pure submodules

$$
(++) \quad Y \subseteq Y^{(1)} \subseteq Y^{(2)} \subseteq \cdots \subseteq Y^{(j)} \subseteq Y^{(j+1)} \subseteq \cdots
$$

of $K_{n-1}$ such that the image of the restriction $d^{(j)}: P\left(\mathcal{Y}^{(j)}\right) \longrightarrow K_{n-1}$ of $d_{n}$ to $P\left(\mathcal{Y}^{(j)}\right)$ contains $Y^{(j)}$ and it is contained in $Y^{(j+1)}$ for $j \geq 1$, and for any finitely generated $R$ module $Z$ and any $R$-homomorphism $f: Z \rightarrow Y^{(j)}$ there exists an $R$-homomorphism $f^{\prime}: Z \longrightarrow P\left(\mathcal{Y}^{(j)}\right)$ such that $f=d^{(j)} f^{\prime}$.

It is clear that the above properties imply Conditions (c1) and (c2) of claim. In view of Lemma 2.6, Condition (c3) is a consequence of (c2), because $Y^{\prime}$ is an $\aleph$-generated pure submodule of $K_{n-1}$.

We construct the sequences $(+)$ and $(++)$ inductively as follows. By applying Lemma 2.5 to the pure submodule $K=K_{n-1}$ of the pure-projective module $P=P\left(I_{n-1}\right)$ and $L=Y$ we get an $\aleph$-generated pure submodule $Y^{\diamond}$ of $K_{n-1}$ containing $Y$. We set $Y^{(1)}=Y^{\diamond}$. By Lemma 2.6, the module $Y^{(1)}$ has a directed union form $Y^{(1)}=\bigcup_{\lambda \in \Omega_{1}} Y_{\lambda}^{(1)}$, where $\Omega_{1}$ is a set of cardinality $\leq \aleph$ and $Y_{\lambda}^{(1)}$ is a countably generated pure-projective pure submodule of $K_{n-1}$ for each $\lambda \in \Omega_{1}$. Since the epimorphism $d_{n}: P\left(I_{n}\right) \longrightarrow K_{n-1}$ is pure and $Y_{\lambda}^{(1)}$ is pure-projective, then for each $\lambda \in \Omega_{1}$ the embedding $v_{\lambda}$ : $Y_{\lambda}^{(1)} \longrightarrow Y^{(1)}$ has a factorisation $v_{\lambda}=d_{n} f_{\lambda}$, where $f_{\lambda} \in \operatorname{Hom}_{R}\left(Y_{\lambda}^{(1)}, P\left(I_{n}\right)\right)$. Since $f_{\lambda}\left(Y_{\lambda}^{(1)}\right)$ is a countably generated submodule of $P\left(I_{n}\right),\left|\Omega_{1}\right| \leq \aleph$ and $\aleph \geq \aleph_{0}$, then there exists a subset $\mathcal{Y}^{(1)}$ of $I_{n}$ of cardinality $\leq \aleph$ containing $\mathcal{Y}$ such that $\sum_{\lambda \in \Omega_{1}} f_{\lambda}\left(Y_{\lambda}^{(1)}\right) \subseteq P\left(\mathcal{Y}^{(1)}\right)$. It follows that the image of the restriction $d^{(1)}: P\left(\mathcal{Y}^{(1)}\right) \longrightarrow K_{n-1}$ of $d_{n}$ to $P\left(\mathcal{Y}^{(1)}\right)$ contains $Y^{(1)} \supset Y$. Moreover, for any finitely generated $R$ module $Z$ and any $R$-homomorphism $f: Z \rightarrow Y^{(1)}$ there exists an $R$-homomorphism $f^{\prime}: Z \longrightarrow P\left(\mathcal{Y}^{(1)}\right)$ such 
that $f=d^{(1)} f^{\prime}$. Indeed, $\operatorname{Im} f$ is a finitely generated submodule of $Y^{(1)}$ and therefore there exists $\lambda \in \Omega_{1}$ such that $\operatorname{Im} f \subseteq Y_{\lambda}^{(1)}$. If we set $f^{\prime}=f_{\lambda} f$, we get the required equality $f=d^{(1)} f^{\prime}$. Hence we conclude $Y^{(1)} \subseteq \operatorname{Im} d^{(1)}$.

Since $\left|\mathcal{Y}^{(1)}\right| \leq \aleph$, the submodule $\operatorname{Im} d^{(1)}$ of $K_{n-1}$ is $\aleph$-generated, and according to Lemma 2.5 there exists an $\aleph$-generated pure submodule $\left(\operatorname{Im} d^{(1)}\right)^{\diamond}$ of $K_{n-1}$ containing $\operatorname{Im} d^{(1)}$. We set $\mathcal{Y}^{(2)}=\left(\operatorname{Im} d^{(1)}\right)^{\diamond}$.

If $j \geq 1$ and $Y^{(j)}, \mathcal{Y}^{(j)}$ are constructed, we construct $Y^{(j+1)}$ and $\mathcal{Y}^{(j+1)}$ by applying the above construction of $Y^{(1)}, \mathcal{Y}^{(1)}$ and $Y^{(2)}$ to $Y^{(j)}$ and the set $\mathcal{Y}^{(j)}$. The details are left to the reader. This finishes the proof of claim.

Now we prove the inductive step. Assume that $n \geq 1$ and that Statements (a1) and (a2) hold for $n-1$. In order to prove (a1) and (a2) for $n$, we assume that $N$ is an $\aleph$-generated submodule of $K_{n}$ and $L$ is an $\aleph$-generated submodule of $K_{0}$. We set $L_{0}=L$. By Lemma 2.5, there exists an $\aleph-$ generated pure submodule $N_{0}^{\diamond_{n}}$ of $P\left(I_{n}\right)$ such that $N \subseteq N_{0}^{\diamond_{n}} \subseteq K_{n}$.

Let $J_{n, 0}^{\prime}$ be a subset of $I_{n}$ of cardinality $\leq \aleph$ such that $N_{0}^{\diamond_{n}} \subseteq P\left(J_{n, 0}^{\prime}\right) \subseteq$ $P\left(I_{n}\right)$. Then the submodule $T_{0}=d_{n}\left(P\left(J_{n, 0}^{\prime}\right)\right)$ of $K_{n-1}=\operatorname{Ker} d_{n-1} \subseteq$ $P\left(I_{n-1}\right)$ is $\aleph$-generated. By applying the induction hypothesis to $T_{0} \subseteq K_{n-1}$ and $L_{0}=L \subseteq K_{0}$ one gets subsets $J_{n-1,0} \subseteq I_{n-1}, \ldots, J_{0,0} \subseteq I_{0}$ of cardinality $\leq \aleph$, an $\aleph$-generated pure submodule $T_{0}^{\diamond_{n-1}} \subseteq K_{n-1}$ of $P\left(J_{n-1,0}\right)$ containing $T_{0}$, an $\aleph$-generated pure submodule $L_{0}^{\diamond_{0}} \subseteq K_{0}$ of $P\left(J_{0,0}\right)$ containing $L_{0}$ such that the sequence

$$
\begin{aligned}
0 \rightarrow T_{0}^{\diamond_{n-1}} \longrightarrow P\left(J_{n-1,0}\right) & \stackrel{d_{n-1,0}}{\longrightarrow} P\left(J_{n-2,0}\right) \\
& \longrightarrow \\
& \cdots P\left(J_{1,0}\right) \stackrel{d_{1,0}}{\longrightarrow} L_{0}^{\diamond_{0}} \rightarrow 0
\end{aligned}
$$

is pure-exact, where $d_{j, 0}$ is the restriction of $d_{j}$ to $P\left(J_{j, 0}\right)$ for $j=1, \ldots, n-1$.

By our claim applied to $Y=T_{0}^{\diamond_{n-1}}$ and $\mathcal{Y}=J_{n, 0}^{\prime}$, there exist a subset $J_{n, 0}$ of $I_{n}$ of cardinality $\leq \aleph$ containing $J_{n, 0}^{\prime}$ and an $\aleph$-generated pure submodule $T_{1}=\left(T_{0}^{\diamond_{n-1}}\right)^{\prime}$ of $K_{n-1}$ containing $T_{0}^{\diamond_{n-1}}$ such that $J_{n, 0}^{\prime} \subseteq J_{n, 0}$, the restriction of $d_{n}$ to $P\left(J_{n, 0}\right)$ yields a pure epimorphism

$$
d_{n, 0}: P\left(J_{n, 0}\right) \longrightarrow T_{1}
$$

and the pure submodule $\operatorname{Ker} d_{n, 0}$ of $P\left(J_{n, 0}\right)$ is $\aleph$-generated. It is clear that $N \subseteq N_{0}^{\diamond_{n}} \subseteq \operatorname{Ker} d_{n, 0}$.

By applying the induction hypothesis to $T_{1} \subseteq K_{n-1}$ and $L_{1}=L_{0}^{\mho_{0}} \subseteq K_{0}$, one gets subsets $J_{n-1,1} \subseteq I_{n-1}, \ldots, J_{0,1} \subseteq I_{0}$ of cardinality $\leq \aleph$, an $\aleph$ generated pure submodule $T_{1}^{\diamond_{n-1}} \subseteq K_{n-1}$ of $P\left(J_{n-1,1}\right)$ containing $T_{1}$, an $\aleph$-generated pure submodule $L_{1}^{\diamond_{0}} \subseteq K_{0}$ of $P\left(J_{0,1}\right)$ containing $L_{1}$ such that 
the sequence

$$
\begin{aligned}
0 \rightarrow T_{1}^{\diamond_{n-1}} \longrightarrow P\left(J_{n-1,1}\right) & \stackrel{d_{n-1,1}}{\longrightarrow} P\left(J_{n-2,1}\right) \\
& \longrightarrow \\
& \longrightarrow P\left(J_{1,1}\right) \stackrel{d_{1,1}}{\longrightarrow} L_{1}^{\diamond_{0}} \rightarrow 0
\end{aligned}
$$

is pure-exact, where $d_{j, 1}$ is the restriction of $d_{j}$ to $P\left(J_{j, 1}\right)$ and $J_{j, 0} \subseteq J_{j, 1} \subseteq I_{j}$ for $j=1, \ldots, n-1$.

By our claim applied to $Y=T_{1}^{\diamond_{n-1}}$ and $\mathcal{Y}=J_{n, 0}$, there exist a subset $J_{n, 1}$ of $I_{n}$ of cardinality $\leq \aleph$ containing $J_{n, 0}$ and an $\aleph$-generated pure submodule $T_{2}=\left(T_{1}^{\diamond_{n-1}}\right)^{\prime}$ of $K_{n-1}$ containing $T_{1}^{\diamond_{n-1}}$ such that the restriction of $d_{n}$ to $P\left(J_{n, 1}\right)$ yields a pure epimorphism

$$
d_{n, 1}: P\left(J_{n, 1}\right) \longrightarrow T_{2},
$$

the submodule Ker $d_{n, 1}$ of $P\left(J_{n, 1}\right)$ is $\aleph$-generated and $N \subseteq N_{0}^{\diamond_{n}} \subseteq \operatorname{Ker} d_{n, 0} \subseteq$ Ker $d_{n, 1}$.

Continuing this way, we construct two sequences

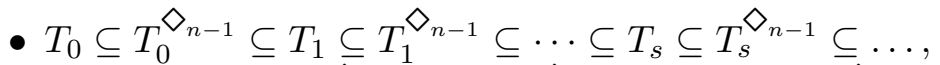

- $L=L_{0} \subseteq L_{1}=L_{0}^{\diamond_{0}} \subseteq L_{2}=L_{1}^{\diamond_{0}} \subseteq \cdots \subseteq L_{s}=L_{s-1}^{\diamond_{0}} \subseteq \ldots$

of $\aleph$-generated submodules of $K_{n-1} \subseteq P\left(I_{n-1}\right)$ and $K_{0} \subseteq P\left(I_{0}\right)$, respectively, and, for each $j \in\{1, \ldots, n\}$, a chain

$$
J_{j, 0} \subseteq J_{j, 1} \subseteq J_{j, 2} \subseteq \cdots \subseteq J_{j, s} \subseteq J_{j, s+1} \subseteq \cdots
$$

of subsets $J_{j, s}$ of $I_{j}$ such that $\left|J_{j, s}\right| \leq \aleph, T_{s}^{\diamond_{n-1}} \subseteq P\left(J_{n-1, s}\right)$ and $L_{s} \subseteq$ $P\left(J_{0, s-1}\right)$ are pure embeddings and the restriction of $d_{n}$ to $P\left(J_{n, s}\right)$ yields a pure epimorphism

$$
d_{n, s}: P\left(J_{n, s}\right) \longrightarrow T_{s+1} .
$$

It follows that, for each $j \in\{1, \ldots, n\}$, there is a chain

$$
P\left(J_{j, 0}\right) \subseteq P\left(J_{j, 1}\right) \subseteq P\left(J_{j, 2}\right) \subseteq \cdots \subseteq P\left(J_{j, s}\right) \subseteq P\left(J_{j, s+1}\right) \subseteq \cdots
$$

of submodules $P\left(J_{j, s}\right)$ of $P\left(I_{j}\right)$, and we get an infinite commutative diagram 


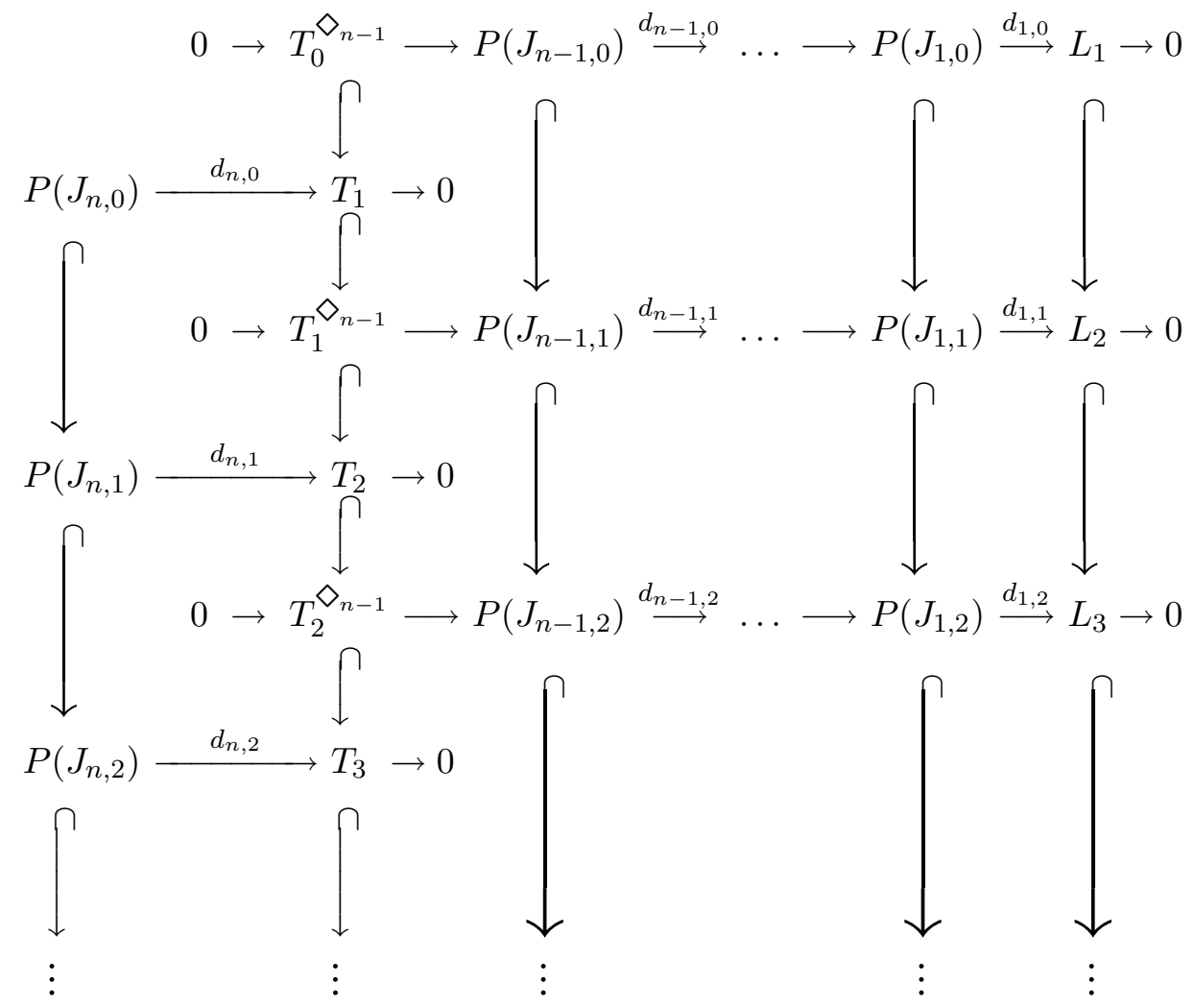

with pure-exact rows, where the vertical homomorphisms are the $R$-module embeddings costructed above. Let

$$
0 \rightarrow N{ }^{\diamond_{n}} \longrightarrow P\left(I_{n}^{\prime}\right) \stackrel{d_{n}^{\prime}}{\longrightarrow} P\left(I_{n-1}^{\prime}\right) \stackrel{d_{n-1}^{\prime}}{\longrightarrow} \ldots \stackrel{d_{2}^{\prime}}{\longrightarrow} P\left(I_{1}^{\prime}\right) \stackrel{d_{1}^{\prime}}{\longrightarrow} L^{\bullet_{0}} \rightarrow 0
$$

be the direct limit of the above system of pure-exact sequences, where

$$
N^{\star}=\bigcup_{s=1}^{\infty} \operatorname{Ker} d_{n, s}, \quad L^{\star 0}=\bigcup_{s=1}^{\infty} L_{s} \quad \text { and } \quad I_{j}^{\prime}=\bigcup_{s=0}^{\infty} J_{j, s}
$$

for $j=1, \ldots, n$. It follows that the limit sequence is pure-exact, consists of $\aleph$-generated modules, $N^{n}=\operatorname{Ker} d_{n}^{\prime}$ is a pure submodule of $P\left(I_{n}^{\prime}\right)$ (and of $K_{n}$ ) containing $N$, the module

$$
\operatorname{Im} d_{n}^{\prime}=\bigcup_{s=1}^{\infty} T_{s}=\bigcup_{s=1}^{\infty} T_{s}^{\diamond_{n-1}}=\operatorname{Ker} d_{n-1}^{\prime}
$$

is a pure submodule of $K_{n-1}$ and $L=\bigcup_{s=1}^{\infty} L_{s}$ is a pure submodule of $P\left(I_{0}^{\prime}\right)$ as well as of $K_{0}$. By Lemma 2.6, the module $N^{n}=\operatorname{Ker} d_{n}^{\prime}$ is $\aleph$-generated. 
This finishes the proof of (a1). The method used in the inductive step of (a1) above also proves the inductive step of (a2). We leave it to the reader. This finishes the proof of (a).

(b) Assume that $n \geq 1$ and $K_{n} \cong K_{0}$. Let $N$ be an $\aleph$-generated submodule of $K_{n}$ and $L$ an $\aleph$-generated submodule of $K_{0}$. Fix an $R$-module isomorphism $f: K_{n} \rightarrow K_{0}$. Keeping the notation above and by applying (a), we construct inductively an infinite commutative diagram

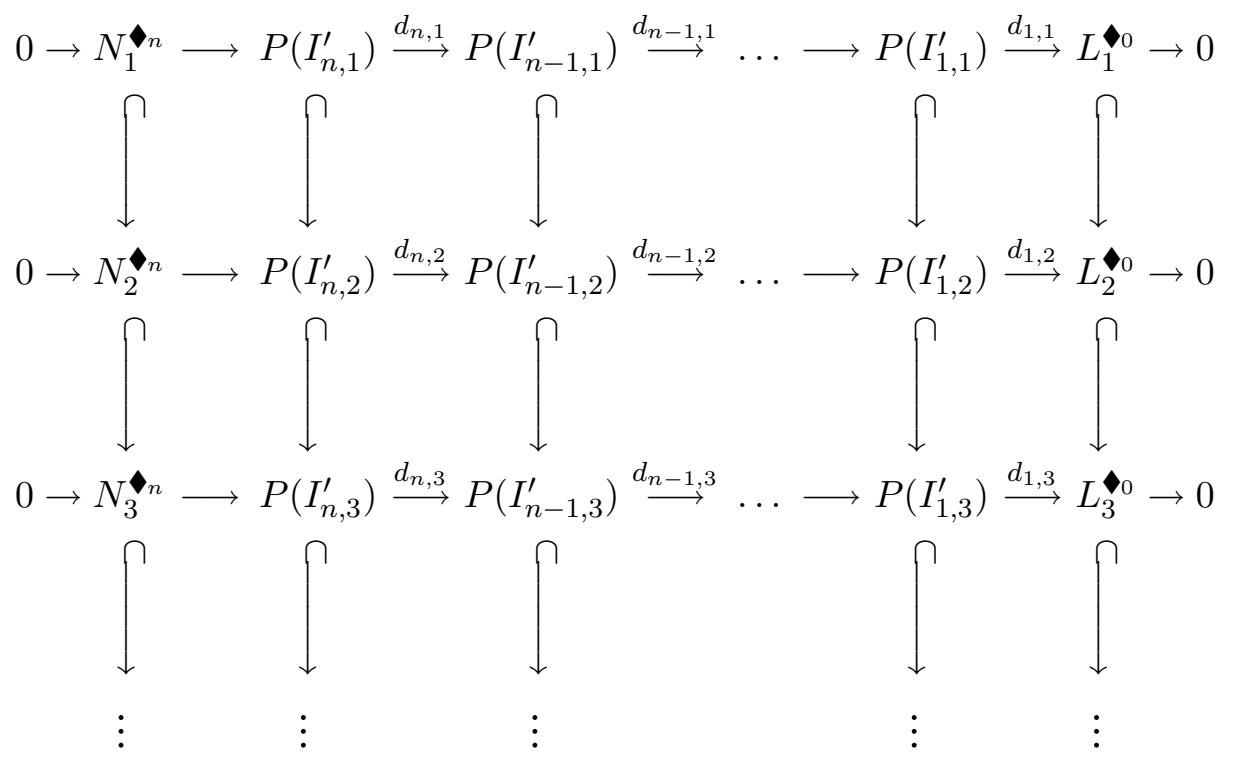

with pure-exact rows, where the vertical homomorphisms are $R$-module embeddings induced by the inclusions $I_{j, 1}^{\prime} \subseteq I_{j, 2}^{\prime} \subseteq I_{j, 3}^{\prime} \subseteq \ldots$, for $j=1, \ldots, n$. We set $N_{1}=N+f^{-1}(L)$ and $L_{1}=f(N)+L$. If the modules $N_{j}, L_{j}$ and $N_{j}^{n}, L_{j}^{0}$ are defined we set

$$
N_{j+1}=N_{j}^{{ }^{n}}+f^{-1}\left(L_{j}^{\bullet^{0}}\right) \quad \text { and } \quad L_{j+1}=f\left(N_{j}^{{ }^{n}}\right)+L_{j}^{{ }^{0}} .
$$

It is clear that $N_{j} \subseteq N_{j}{ }^{n} \subseteq N_{j+1}, L_{j} \subseteq L_{j} \subseteq L_{j+1}, f\left(N_{1}\right)=L_{1}$ and, for each $j \geq 1$, we get $f\left(N_{j+1}\right)=L_{j+1}$. Let

$$
0 \rightarrow N \bullet P\left(I_{n}^{\prime}\right) \stackrel{d_{n}^{\prime}}{\longrightarrow} P\left(I_{n-1}^{\prime}\right) \stackrel{d_{n-1}^{\prime}}{\longrightarrow} \ldots \stackrel{d_{2}^{\prime}}{\longrightarrow} P\left(I_{1}^{\prime}\right) \stackrel{d_{1}^{\prime}}{\longrightarrow} L \bullet 0
$$

be the direct limit of the above system of pure-exact sequences, where

$$
N^{\star}=\bigcup_{s=1}^{\infty} N_{s}^{n}=\bigcup_{s=1}^{\infty} N_{s}, \quad L=\bigcup_{s=1}^{\infty} L_{s}=\bigcup_{s=1}^{\infty} L_{s} \quad \text { and } \quad I_{j}^{\prime}=\bigcup_{s=0}^{\infty} I_{j, s}^{\prime}
$$

for $j=1, \ldots, n$. It is easy to see that $f(N)=L$. Thus the modules $N, L$ are isomorphic and the statement (b) follows. This finishes the proof. 
The claim proved in the above proof yields the following useful result.

Corollary 3.2. Assume that $\aleph$ is an infinite cardinal number, $h: P \rightarrow K$ is a pure epimorphism in $\operatorname{Mod}(R), P$ is an $\aleph$-generated pure-projective module and $K$ is a pure submodule of a pure-projective module. For any $\aleph$-generated submodule $Y$ of $K$ and any subset $\mathcal{X}$ of $P$ of cardinality $\leq \aleph$ there exist an $\aleph$-generated direct summand $P^{\prime}$ of $P$ containing $\mathcal{X}$ and an $\aleph$-generated pure submodule $Y^{\prime}$ of $K$ containing $Y$ such that $h\left(P^{\prime}\right)=Y^{\prime}$, the restriction $h^{\prime}: P^{\prime} \rightarrow Y^{\prime}$ of $h$ to $P^{\prime}$ is a pure epimorphism and the module Ker $h^{\prime}$ is $\aleph$-generated.

Proof. Let $h: P \rightarrow K$ be a pure-epimorphism. By our assumption, $K$ is a pure submodule of a pure-projective module $P_{0}$. We apply the claim in the proof above to $n=1, P_{1}=P, d_{1}=h, M=P_{0} / K$ and $d_{0}: P_{0} \rightarrow M$ the canonical epimorphism. By Kaplansky theorem [13], the module $P$ has the form $P_{1}=P\left(I_{1}\right)=\bigoplus_{t \in I_{1}} Q_{t}$, where $Q_{t}$ is a countably generated pureprojective module for $t \in I_{1}$, in the notation introduced above. Since $\mathcal{X}$ is a subset of $P$ of cardinality $\leq \aleph$ there exist a subset $\mathcal{Y}$ of $I_{1}$ of cardinality $\leq \aleph$ containing $\mathcal{X}$. Then the corollary is an immediate consequence of Statements (c1), (c2) and (c3) of the claim.

One of the main results of this paper is the following theorem describing a pure-projective structure of pure-syzygy modules of any $R$-module (compare with [23, Theorem 1.5]).

Theorem 3.3. Let $R$ be a ring, $M$ a right $R$-module and

$$
\ldots \longrightarrow P_{n} \stackrel{d_{n}}{\longrightarrow} P_{n-1} \stackrel{d_{n-1}}{\longrightarrow} \ldots \stackrel{d_{2}}{\longrightarrow} P_{1} \stackrel{d_{1}}{\longrightarrow} P_{0} \stackrel{d_{0}}{\longrightarrow} M \rightarrow 0
$$

a pure-exact sequence in $\operatorname{Mod}(R)$ such that the modules $P_{0}, \ldots, P_{n}, \ldots$ are pure-projective. Then, for each $n \geq 0$, the nth pure-syzygy module Ker $d_{n}$ of $M$ is an $\aleph_{n}$-directed union of $\aleph_{n}$-generated pure-projective pure $R$-submodules of $\operatorname{Ker} d_{n}$, which are pure submodules of $P_{n}$.

Proof. Fix $n \geq 0$ and consider the $n$th pure-syzygy submodule $K_{n}=\operatorname{Ker} d_{n}$ of $P_{n}$. Set $K_{0}=\operatorname{Ker} d_{0}$. It is sufficient to show that any $\aleph_{n}$-generated submodule $N$ of $K_{n}$ is a submodule of an $\aleph_{n}$-generated pure-projective submodule of $K_{n}$, which is pure in $K_{n}$. Let $N$ be an $\aleph_{n}$-generated submodule of $K_{n}$. By applying Proposition 3.1 to $N \subseteq K_{n}$ and to the submodule $L=(0)$ of $K_{0}$, one gets a pure-exact sequence

$$
0 \rightarrow N^{\bullet} \longrightarrow P_{n}^{\prime} \stackrel{d_{n}^{\prime}}{\longrightarrow} P_{n-1}^{\prime} \stackrel{d_{n-1}^{\prime}}{\longrightarrow} \ldots \stackrel{d_{2}^{\prime}}{\longrightarrow} P_{1}^{\prime} \stackrel{d_{1}^{\prime}}{\longrightarrow} L{ }^{\bullet} \rightarrow 0
$$

consisting of $\aleph_{n}$-generated modules, where $P_{n}^{\prime}, \ldots, P_{1}^{\prime}$ are pure-projective modules, $N$ is a pure submodule of $K_{n}, L$ is a pure submodule of $P_{0}$ and $N \subseteq N{ }^{n}$. In case $n=0$ we get just $N{ }^{\star}=L$. 
Let $P_{0}^{\prime \prime}$ be a right $R$-module such that the module $P^{\prime}=P_{0} \oplus P_{0}^{\prime \prime}$ is a direct sum of finitely presented modules. Since $L$ is a pure submodule of $P_{0}$, it is a pure submodule of $P^{\prime}$ and therefore $L^{\circ}$ is a pure submodule of an $\aleph_{n}$-generated pure-projective direct summand $P$ of $P_{0}$. Then the module $P / L{ }^{*}$ is $\aleph_{n}$-presented and Proposition 2.2 yields

$$
\text { P.pd } P / L \leq n+1 \text {. }
$$

It follows that the submodule $N_{n}$ of $P_{n}^{\prime}$ in the pure-exact sequence (3.4) is pure-projective, because $P_{n}^{\prime}, \ldots, P_{1}^{\prime}$ are pure-projective modules. This finishes the proof.

As a consequence of Theorem 3.3 we get the following structure theorem on syzygy modules of flat modules proved by the author in [23, Theorem $1.5]$.

Corollary 3.5. Let $R$ be a ring, $M$ a right flat $R$-module and $n \geq 0$ an integer. If

$$
0 \rightarrow K_{n} \longrightarrow F_{n} \longrightarrow F_{n-1} \longrightarrow \ldots \longrightarrow F_{1} \longrightarrow F_{0} \longrightarrow M \rightarrow 0
$$

is an exact sequence in $\operatorname{Mod}(R)$ such that the modules $F_{0}, \ldots, F_{n}$ are projective, then the $n$-th syzygy module $K_{n}$ of $M$ is an $\aleph_{n}$-directed union of $\aleph_{n}$-generated projective pure $R$-submodules of $K_{n}$ (which are pure submodules of $\left.F_{n}\right)$.

Proof. Since $M$ is flat then the sequence (3.6) is pure-exact and the projective modules $F_{0}, \ldots, F_{n}$ are obviously pure-projective. It follows from Theorem 3.3 that $K_{n}$ is an $\aleph_{n}$-directed union of $\aleph_{n}$-generated pure-projective $R$-submodules $U_{\mu}$ of $K_{n}$, with $\mu \in \Omega_{n}$, which are pure submodules of the projective module $F_{n}$. Hence each of the modules $U_{\mu}$ is flat and therefore any epimorphism $h_{\mu}: P_{\mu} \rightarrow U_{\mu}$ from a projective module $P_{\mu}$ to $U_{\mu}$ is a pure epimorphism. Since the module $U_{\mu}$ is pure-projective, then the epimorphism $h_{\mu}$ splits and, consequently, the module $U_{\mu}$ is projective for any $\mu \in \Omega_{n}$. This completes the proof.

\section{Pure-periodic modules.}

Definition 4.1. Let $R$ be a ring. A right $R$-module $T$ is defined to be pure-periodic of period $p \geq 1$ if there exists a pure-exact sequence

$$
0 \rightarrow T \longrightarrow P_{p} \longrightarrow P_{p-1} \longrightarrow \ldots \longrightarrow P_{1} \longrightarrow T \rightarrow 0
$$

in $\operatorname{Mod}(R)$ such that the modules $P_{1}, \ldots, P_{p}$ are pure-projective.

We start with two useful lemmata on pure-periodic modules.

Lemma 4.2. Assume that $T$ is a pure-periodic right $R$-module of period $p \geq 1$ and $\aleph$ is an infinite cardinal number. Then, for any $\aleph$-generated 
submodule $U$ of $T$, there exists an $\aleph$-generated pure submodule $U$ of $T$ such that $U \subseteq U$ and $U$ is pure-periodic of the same period $p$.

Proof. Since $T$ is pure-periodic of period $p \geq 1$, then $T$ is a pure submodule of a pure-projective module $P_{0}$ and there exists a pure-exact sequence

$$
0 \rightarrow K_{p} \longrightarrow P_{p} \stackrel{d_{p}}{\longrightarrow} P_{p-1} \stackrel{d_{p-1}}{\longrightarrow} \ldots \stackrel{d_{2}}{\longrightarrow} P_{1} \stackrel{d_{1}}{\longrightarrow} P_{0} \stackrel{d_{0}}{\longrightarrow} M \rightarrow 0
$$

where $M=P_{0} / T, d_{0}$ is the canonical projection, Ker $d_{0}=T \cong K_{p}=\operatorname{Ker} d_{p}$ and the modules $P_{1}, \ldots, P_{p}$ are pure-projective. Let $f: T \rightarrow K_{p}$ be an $R$ module isomorphism and let $N=f(U)$. Then Proposition 3.1 (b) applies to the above sequence with $K_{0}=T$ and $N \cong L=U$. Consequently, there exists an $\aleph$-generated pure submodule $U$ of $T$ satisfying the required conditions.

Lemma 4.3. Assume that $\aleph \geq \aleph_{1}$ is an infinite cardinal number and $T$ an $\aleph$-generated pure submodule of a pure-projective right $R$-module.

(a) The module $T$ is a continuous well-ordered union of pure submodules $T_{\xi}$, with $\xi<\gamma$, such that, for each $\xi$, the module $T_{\xi}$ is generated by a set of cardinality $<\aleph$.

(b) If, in addition, $T$ is pure-periodic of period $p \geq 1$ then, for each $\xi<\gamma$, the pure submodule $T_{\xi}$ of $T$ can be chosen pure-periodic of the same period $p$.

Proof. Let $\aleph^{\prime}<\aleph$ be an infinite cardinal. It follows from Lemma 2.5 that any $\aleph^{\prime}$-generated submodule $X$ of $T$ can be embedded in an $\aleph^{\prime}$-generated pure submodule $X^{\diamond}$ of $T$. If, in addition, $T$ is pure-periodic of period $p \geq 1$ then, according to Lemma $4.2, X$ can be embedded in an $\aleph^{\prime}$-generated pureperiodic pure submodule $X$ of $T$ of the same period $p$.

It is well-known that $T$ can be represented as a continuous well-ordered union of submodules $U_{\xi}$, with $\xi<\gamma$, such that, for each $\xi$, the module $U_{\xi}$ is generated by a set of cardinality $<\aleph$ (see [11, Lemme 1.4] and [19, Lemma $2.2]$ ). Let us define a transfinite increasing chain of pure-submodules $T_{\xi}$ of $T$, with $\xi<\gamma$, having $<\aleph$ generators as follows (compare with $[\mathbf{1 9}$, Theorem 1.5] and [16, Lemma 1.7]). We set $T_{0}=(0)$ and $T_{1}=U_{1}^{\diamond}$. If $T_{\xi}$ is defined and $\xi+1<\gamma$, we set $T_{\xi+1}=\left(T_{\xi}+U_{\xi+1}\right)^{\diamond}$. Finally, we set $T_{\tau}=\bigcup_{\xi<\tau} T_{\xi}$ if $\tau$ is a limit ordinal number. This proves Statement (a).

In order to prove (b), we assume that $T$ is pure-periodic of period $p \geq 1$. It follows that $T$ is a pure submodule of a pure-projective module $P_{0}$ and there exists a pure-exact sequence

$$
0 \rightarrow K_{p} \longrightarrow P_{p} \stackrel{d_{p}}{\longrightarrow} P_{p-1} \stackrel{d_{p-1}}{\longrightarrow} \ldots \stackrel{d_{2}}{\longrightarrow} P_{1} \stackrel{d_{1}}{\longrightarrow} P_{0} \stackrel{d_{0}}{\longrightarrow} M \rightarrow 0,
$$

where $M=P_{0} / T, d_{0}$ is the canonical projection, Ker $d_{0}=T \cong K_{p}=\operatorname{Ker} d_{p}$ and the modules $P_{1}, \ldots, P_{p}$ are pure-projective. Let $f: T \rightarrow K_{p}$ be an $R$ module isomorphism and let $N=f(U)$. 
In the notation introduced in (a1) and (a2) of the proof of Proposition 3.1 with $n=p$, we set $K_{p}=\operatorname{Ker} d_{p} \cong K_{0}=T, P_{j}=P\left(I_{j}\right)$ for $j=0,1, \ldots p$. We define a transfinite increasing chain of pure-periodic pure-submodules $T_{\xi}$ of $T$ generated by sets of cardinality $<\aleph$, a transfinite chain of subsets $I_{j, \xi}$ of $I_{j}$ of cardinality $\left|I_{j, \xi}\right|<\aleph$ for $\xi<\gamma$ and $j=0,1, \ldots p$, such that $I_{j, \xi} \subseteq I_{j, \xi+1}$ and there is a commutative diagram

$(*) \xi$

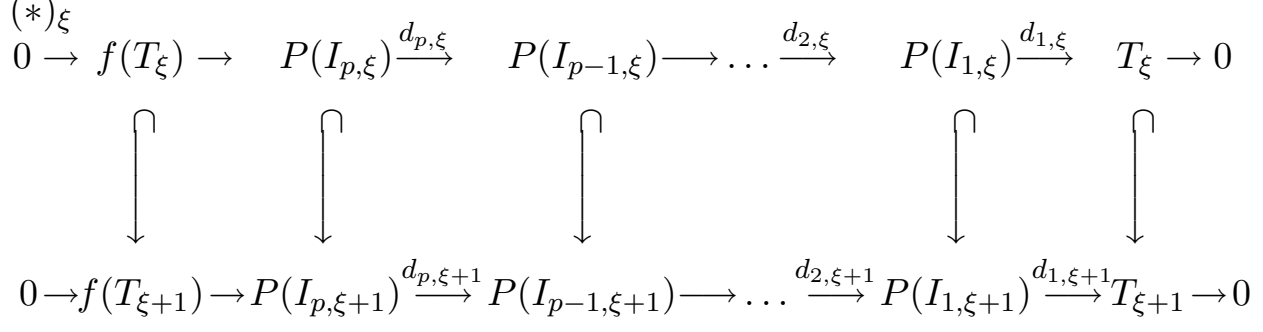

with pure-exact rows, where the vertical arrows are natural embeddings induced by the inclusions $I_{j, \xi} \subseteq I_{j, \xi+1}$ for $j=1, \ldots, p$ and the module $T_{\xi}$ is a pure submodule of $P\left(I_{0, \xi}\right)$.

We set $T_{0}=(0)$ and $I_{j, 0}=\emptyset$ for $j=0,1, \ldots p$. If $T_{\xi}, I_{j, \xi}$ and a corresponding pure-exact sequence are defined, we set $T_{\xi+1}=\left(T_{\xi}+U_{\xi+1}\right)$ and we define the sets $I_{p, \xi+1}, I_{p-1, \xi+1}, \ldots, I_{0, \xi+1}$ by applying Proposition 3.1 (b) and its proof in such a way that the above diagram $(*)_{\xi}$ is commutative, the rows are pure-exact and the module $T_{\xi}$ in $(*)_{\xi}$ is a pure submodule of $P\left(I_{0, \xi}\right)$ generated by a set of cardinality $<\aleph$ and the module $f\left(T_{\xi}\right)$ in $(*)_{\xi}$ is a pure submodule of $P\left(I_{p, \xi}\right)$.

If $\tau$ is a limit ordinal number we set $T_{\tau}=\bigcup_{\xi<\tau} T_{\xi}$ and $I_{j, \tau}=\bigcup_{\xi<\tau} I_{j, \xi}$. Then the direct limit of the sequences $(*)_{\xi}$ is a pure-exact sequence

$$
\begin{aligned}
0 \rightarrow f\left(T_{\tau}\right) \rightarrow P\left(I_{p, \tau}\right) \stackrel{d_{p, \tau}}{\longrightarrow} P\left(I_{p-1, \tau}\right) & \stackrel{d_{p-1, \tau}}{\longrightarrow} \ldots \\
& \stackrel{d_{2, \tau}}{\longrightarrow} P\left(I_{1, \tau}\right) \stackrel{d_{1, \tau}}{\longrightarrow} T_{\tau} \rightarrow 0
\end{aligned}
$$

and the required conditions are satisfied. This finishes the proof.

Now we are able to prove main results of this section. The following theorem implies Theorem 1.3:

Theorem 4.4. Let $R$ be a ring. Every pure-periodic $R$-module is pureprojective.

Proof. Assume that $T$ is a pure-periodic right $R$-module of period $p \geq 1$, and $T$ is generated by a set of cardinality $\leq \aleph$. We prove the theorem by transfinite induction on $\aleph$.

First, we suppose that $\aleph=\aleph_{n}$, where $n \geq 0$. Since $T$ is pure-periodic, it follows that $T$ is a pure submodule of a pure-projective module $P_{0}$ and 
there exists a pure-exact sequence

$$
0 \rightarrow \operatorname{Ker} d_{p} \longrightarrow P_{p} \stackrel{d_{p}}{\longrightarrow} P_{p-1} \longrightarrow \ldots \longrightarrow P_{1} \stackrel{d_{1}}{\longrightarrow} P_{0} \longrightarrow M \rightarrow 0
$$

such that the modules $P_{0}, \ldots, P_{p}$ are pure-projective, $M=P_{0} / T$ and $T=$ $\operatorname{Im} d_{1}$. Without loss of generality we can suppose that $p \geq n$. In that case $T$ is also $\aleph_{p}$-generated, and therefore we can assume $p=n$.

By Theorem 3.3, the $p$ th pure-syzygy module Ker $d_{p} \cong T$ of $P_{p}$ is an $\aleph_{p^{-}}$ directed union of $\aleph_{p}$-generated pure-projective pure submodules. It follows that $T$ is pure-projective, because it is $\aleph_{p}$-generated.

Next, we assume that $T$ is an arbitrary $\aleph$-generated pure-periodic $R$ module, $\aleph>\aleph_{n}$ for all integers $n \geq 1$, and the theorem holds for all cardinals smaller than $\aleph$. By Lemma 4.3, $T$ is a continuous well-ordered union of pureperiodic pure submodules $T_{\xi}, \xi<\gamma$, such that for each $\xi<\gamma$ the module $T_{\xi}$ is generated by a set of cardinality $<\aleph$. By the inductive hypothesis, each of the modules $T_{\xi}$ is pure-projective and therefore P.pd $T_{\xi+1} / T_{\xi} \leq 1$. It follows from Proposition 2.1 that P.pd $T \leq 1$. Hence the submodule Ker $d_{2}$ of $P_{2}$ in the pure-exact sequence above is pure-projective and consequently the monomorphism $T \cong \operatorname{Ker} d_{p} \hookrightarrow P_{p}$ splits. This shows that $T$ is pureprojective and finishes the proof.

Corollary 4.5. Let $M$ be a right $R$-module and

$$
\ldots \longrightarrow P_{n} \stackrel{d_{n}}{\longrightarrow} P_{n-1} \stackrel{d_{n-1}}{\longrightarrow} \ldots \stackrel{d_{2}}{\longrightarrow} P_{1} \stackrel{d_{1}}{\longrightarrow} P_{0} \stackrel{d_{0}}{\longrightarrow} M \rightarrow 0
$$

a pure-exact sequence, where the modules $P_{0}, \ldots, P_{n}, \ldots$ are pure-projective. Assume that there exist two integers $n \geq 0$ and $p \geq 1$ such that Ker $d_{n+p} \cong$ Ker $d_{n}$. Then P.pd $M \leq n+1$.

Proof. By our assumption, the $n$th pure syzygy module Ker $d_{n}$ is pureperiodic of period $p \geq 1$. It then follows from Theorem 4.4 that the module Ker $d_{n}$ is pure-projective and, consequently, P.pd $M \leq n+1$.

As a consequence of Theorem 4.4 we get the following form of [5, Theorem $2.5]$ :

Corollary 4.6. Let $R$ be a ring, $M$ a right flat $R$-module and $n \geq 1$ an integer. If there exists an exact sequence

$$
0 \rightarrow M \longrightarrow F_{n} \longrightarrow F_{n-1} \longrightarrow \ldots \longrightarrow F_{1} \longrightarrow F_{0} \longrightarrow M \rightarrow 0
$$

in $\operatorname{Mod}(R)$ such that the modules $F_{0}, \ldots, F_{n}$ are projective, then $M$ is projective.

Proof. Since $M$ is flat, the sequence (4.7) is pure-exact and the projective modules $F_{0}, \ldots, F_{n}$ are obviously pure-projective. It follows that $M$ is pure-periodic and, according to Theorem 4.4, the sequence (4.7) splits. Consequently, $M$ is projective. 
We finish this section with the following interesting question suggested by referee and related with the problems studied in [4] and [5].

Problem 4.8. Assume that $R$ is an associative ring with an identity element and $H$ is a subgroup of finite index in a group $G$. Let $M$ be an arbitrary right module over the group ring $R G$. Is the $R G$-module $M$ pure-projective, if $M$ is pure-projective, when viewed as an $R H$-module?

\section{A structure of pure-projective resolutions in Grothendieck categories.}

We show in this section that the main results of Sections 3 and 4 on puresyzygies and pure-periodic modules generalize from the module category $\operatorname{Mod}(R)$ to an arbitrary locally finitely presented Grothendieck category $\mathcal{A}$ (see $[21])$.

Throughout we denote by $\mathcal{A}$ a locally finitely presented Grothendieck category. We recall that an object $L$ of $\mathcal{A}$ is said to be finitely presented if the additive functor $\operatorname{Hom}_{\mathcal{A}}(L,-): \mathcal{A} \longrightarrow \mathcal{A} b$ commutes with filtered direct limits (see $[\mathbf{2 1}],[\mathbf{2 6}],[\mathbf{3 0}]$ ). A long exact sequence $\cdots \rightarrow X_{n-1} \rightarrow$ $X_{n} \rightarrow X_{n+1} \rightarrow \ldots$ in $\mathcal{A}$ is said to be pure if the induced sequence $\cdots \rightarrow$ $\operatorname{Hom}_{\mathcal{A}}\left(L, X_{n-1}\right) \rightarrow \operatorname{Hom}_{\mathcal{A}}\left(L, X_{n}\right) \rightarrow \operatorname{Hom}_{\mathcal{A}}\left(L, X_{n+1}\right) \rightarrow \ldots$ of abelian groups is exact for any finitely presented object $L$. An epimorphism $f: Y \rightarrow$ $Z$ in $\mathcal{A}$ is said to be pure if the exact sequence $0 \longrightarrow \operatorname{Ker} f \longrightarrow Y \stackrel{f}{\longrightarrow} Z \longrightarrow 0$ is pure. A subobject $X$ of $Y$ in $\mathcal{A}$ is said to be pure if the exact sequence $0 \rightarrow X \rightarrow Y \rightarrow Y / X \rightarrow 0$ is pure. An object $P$ in $\mathcal{A}$ is said to be pureprojective if for any pure-epimorphism $f: Y \rightarrow Z$ in $\mathcal{A}$ the induced group homomorphism $\operatorname{Hom}_{\mathcal{A}}(P, f): \operatorname{Hom}_{\mathcal{A}}(P, Y) \longrightarrow \operatorname{Hom}_{\mathcal{A}}(P, Z)$ is surjective.

It is well-known that:

- An object $P$ in locally finitely presented Grothendieck category $\mathcal{A}$ is pure-projective if and only if $P$ is a direct summand of a coproduct of finitely presented objects.

- Every object $M$ in $\mathcal{A}$ admits a pure-projective pure resolution in $\mathcal{A}$, that is, there is a pure-exact sequence

$$
\ldots \longrightarrow P_{n} \stackrel{d_{n}}{\longrightarrow} P_{n-1} \stackrel{d_{n-1}}{\longrightarrow} \ldots \stackrel{d_{2}}{\longrightarrow} P_{1} \stackrel{d_{1}}{\longrightarrow} P_{0} \stackrel{d_{0}}{\longrightarrow} M \rightarrow 0,
$$

where $P_{0}, \ldots, P_{n}, \ldots$ are pure-projective objects (see [26], [30]).

Then, for each $n \geq 0$, the functor $\operatorname{Pext}_{\mathcal{A}}^{n}: \mathcal{A}^{o p} \times \mathcal{A} \longrightarrow \mathcal{A} b$ is naturally defined and, for any object $M$ in $\mathcal{A}$, the pure-projective dimension P.pd $M$ of $M$ is defined in a natural way (see [30] and [26]).

The main results of Sections 3 and 4 extend to the context of locally finitely presented Grothendieck categories as follows:

Theorem 5.2. Let $M$ be an arbitrary object of a locally finitely presented Grothendieck category $\mathcal{A}$. If (5.1) is a pure-exact sequence in $\mathcal{A}$ such that 
the objects $P_{0}, \ldots, P_{n}, \ldots$ are pure-projective, then for each $n \geq 0$ the $n$ th pure-syzygy object $\operatorname{Ker} d_{n}$ of $M$ is an $\aleph_{n}$-directed union of $\aleph_{n}$-generated pure-projective objects, which are pure subobjects of $P_{n}$.

Theorem 5.3. Let $M$ be an object of a locally finitely presented Grothendieck category $\mathcal{A}$. If there exists a pure-exact sequence

$$
0 \rightarrow M \longrightarrow P_{n} \longrightarrow P_{n-1} \longrightarrow \ldots \longrightarrow P_{1} \longrightarrow P_{0} \longrightarrow M \rightarrow 0
$$

in $\mathcal{A}$ such that the objects $P_{0}, \ldots, P_{n}$ are pure-projective, then $M$ is pureprojective.

In other words, every pure-periodic object $M$ of $\mathcal{A}$ is pure-projective.

\section{Outline of the proof.}

First we note that Proposition 2.1 (a pure version of a theorem of Auslander $[\mathbf{2}]$ ) extends to an arbitrary Grothendieck category $\mathcal{A}$ (see [25] and [26, Proposition 2.6]). Further, by [26, Theorem 2.12], if $M$ is an $\aleph_{n}$-presented object of a locally finitely presented Grothendieck category $\mathcal{A}$ and $n \geq 0$, then P.pd M $\leq n+1$. Finally, we note that the proof of Lemma 2.5 uses only categorical arguments and therefore extends to our situation. It then follows that also our Lemma 2.6 remains valid with $\operatorname{Mod}(R)$ and $\mathcal{A}$ interchanged. Since the Kaplansky theorem [13] also remains valid for objects of a Grothendieck category $\mathcal{A}$ (see [21]) then the proof of Proposition 3.1 works with $\operatorname{Mod}(R)$ and $\mathcal{A}$ interchanged. Thus, applying the arguments in the proof of Theorems 3.3 and 4.4, we easily get Theorems 5.2 and 5.3. The details are left to the reader.

Acknowledgment. The author is indebted to the referee for useful remarks, comments and improvements.

\section{References}

[1] F.W. Anderson and K.R. Fuller, Rings and Categories of Modules, Springer-Verlag, 1991, MR 94i:16001, Zbl 0765.16001.

[2] M. Auslander, On the dimension of modules and algebras III. Global dimensions, Nagoya J. Math., 9 (1955), 66-77, MR 17,579a, Zbl 0067.27103.

[3] S. Balcerzyk, On projective dimension of direct limit of modules, Bull. Acad. Polon. Sci., Sér. Sci. Math., 14 (1966), 241-244, MR 35 \#2946, Zbl 0158.28902.

[4] D.J. Benson, Flat modules over group rings of finite groups, Algebras and Representation Theory, 2 (1999), 287-294, MR 2000g:20013, Zbl 0938.20003.

[5] D.J. Benson and K.R. Goodearl, Periodic flat modules, and flat modules for finite groups, Pacific J. Math., 196 (2000), 45-67, MR 2002c:20006.

[6] P.M. Cohn, On the free product of associative rings, Math. Z., 71 (1959), 380-398, MR 21 \#5648, Zbl 0087.26303.

[7] P. Griffith, On the decomposition of modules and generalized left uniserial rings, Math. Ann., 184 (1970), 300-308, MR 41 \#1790, Zbl 0184.06404. 
[8] L. Gruson and C.U. Jensen, L-dimension of rings and modules, in 'Lecture Notes in Math., 867,' Springer-Verlag, Berlin-Heidelberg-New York-Tokyo, 1980, 234-294.

[9] B. Huisgen-Zimmermann, Purity, algebraic compactness, direct sum decompositions, and representation type, in 'Infinite Length Modules' (Eds. H. Krause and C.M. Ringel), Trends in Mathematics, Birkäuser Verlag, Basel-Boston-Berlin, 2000, 331367, MR 2002c:16008.

[10] C.U. Jensen, On homological dimensions of rings with countably generated ideals, Mat. Scand., 18 (1966), 97-105, MR 34 \#7611, Zbl 0145.26605.

[11] _ Les Foncteurs Dérivés de lim et leurs Applications en Théorie des Modules, Lecture Notes in Math., 254, Springer-Verlag, Berlin-Heidelberg-New York, 1972, MR 53 \#10874, Zbl 0238.18007.

[12] C.U. Jensen and H. Lenzing, Model Theoretic Algebra With Particular Emphasis on Fields, Rings, Modules, Algebra, Logic and Applications, Vol. 2, Gordon \& Breach Science Publishers, New York-London, 1989, MR 91m:03038, Zbl 0728.03026.

[13] I. Kaplansky, Projective modules, Annals of Math., 68 (1958), 372-377, MR 20 \#6453, Zbl 0083.25802.

[14] R. Kiełpiński, O Charakteryzacji i Strukturze Modutów $\mathbb{P}$-serwantnie Injektywnych, Doctoral Thesis, Toruń, 1967 (in Polish).

[15] _ On Г-pure injective modules, Bull. Polon. Acad. Sci., Ser. Math., 15 (1967), 127-131, MR 36 \#3816, Zbl 0159.04602.

[16] R. Kiełpiński and D. Simson, On pure homological dimension, Bull. Polon. Acad. Sci., Ser. Math., 23 (1975), 1-6, MR 53 \#10872, Zbl 0303.16016.

[17] D. Lazard, Autour de la platitude, Bull. Soc. Math. France, 97 (1969), 81-128, MR 40 \#7310, Zbl 0174.33301.

[18] B. Mitchell, Rings with several objects, Advances in Math., 8 (1972), 1-161, MR 45 \#3524, Zbl 0232.18009.

[19] B. Osofsky, Upper bounds on homological dimensions, Nagoya J. Math., 32 (1968), 315-322, MR 38 \#1128, Zbl 0162.05102.

[20] _ Projective dimensions of nice directed unions, J. Pure Appl. Algebra, 13 (1978), 179-219, MR 81d:16019, Zbl 0392.16023.

[21] N. Popescu, Abelian Categories With Applications to Rings and Modules, Academic Press, 1973, MR 49 \#5130, Zbl 0271.18006.

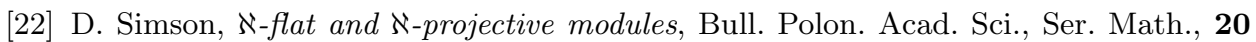
(1972), 109-114, MR 47 \#3450, Zbl 0232.16021.

[23] _ On projective resolutions of flat modules, Colloq. Math., 29 (1974), 209-218, MR 50 \#13147, Zbl 0279.16013.

[24] _ A remark on projective dimensions of flat modules, Math. Annalen, 209 (1974), 181-182, MR 49 \#9031, Zbl 0285.16024.

[25] _ On colimits of injectives in Grothendieck categories, Arkiv för Mat., 12 (1974), 161-165, MR 51 \#652, Zbl 0297.18003.

[26] _ On pure global dimension of locally finitely presented Grothendieck categories, Fund. Math., 96 (1977), 91-116, MR 58 \#845, Zbl 0361.18010.

[27] _ Pure semisimple categories and rings of finite representation type, J. Algebra, 48 (1977), 290-296, MR 57 \#380, Zbl 0409.16030; Corrigendum, 67 (1980), 254-256, MR 82d:16028, Zbl 0449.16027. 
[28] _ A class of potential counter-examples to the pure semisimplicity conjecture, Proc. Conf. 'Model Theory and Modules', Essen, 1994; Algebra, Logic and Applications, Vol. 9, Gordon \& Breach Science Publishers, Amsterdam, 1997, 345-373, MR 2000d:16019, Zbl 0936.16010.

[29] B. Stenström, Pure submodules, Arkiv för Mat., 7 (1967), 159-171, MR 36 \#6473, Zbl 0153.34302.

[30] _ Purity in functor categories, J. Algebra, 8 (1968), 352-361, MR 37 \#5271, Zbl 0157.04204.

[31] R.B. Warfield, Purity and algebraic compactness for modules, Pacific J. Math., 28 (1969), 699-719, MR 39 \#4212, Zbl 0172.04801.

Received February 12, 2001 and revised February 26, 2002. This author was partially supported by Polish KBN Grant 5 P0 3A 01521.

FACUlty of Mathematics and Informatics

NiChOlas COPERNICUS UNIVERSITY

ul. Chopina 12/18

87-100 Toruń, Poland

E-mail address: simson@mat.uni.torun.pl 